\title{
Diacronie
}

Studi di Storia Contemporanea

$N^{\circ} 24,4 \mid 2015$

Le dittature militari: fisionomia ed eredità politica

\section{Le conseguenze del golpe del 1964 sul movimento sindacale brasiliano}

\section{Alejandra Estevez e Marco Antônio Teixeira}

Traduttore: Jacopo Bassi

\section{OpenEdition \\ Journals}

Edizione digitale

URL: http://journals.openedition.org/diacronie/3684

DOI: $10.4000 /$ diacronie.3684

ISSN: 2038-0925

Editore

Association culturelle Diacronie

Notizia bibliografica digitale

Alejandra Estevez e Marco Antônio Teixeira, « Le conseguenze del golpe del 1964 sul movimento sindacale brasiliano », Diacronie [Online], № 24, 4 | 2015, documento 6, Messo online il 29 décembre 2015, consultato il 19 avril 2019. URL : http://journals.openedition.org/diacronie/3684 ; DOI : 10.4000/ diacronie.3684 


\title{
Diacronie
}

6/

\section{Le conseguenze del golpe del 1964 sul movimento sindacale brasiliano}

\author{
Alejandra ESTEVEZ, Marco Antônio TEIXEIRA* \\ traduzione a cura di Jacopo BASSI
}

L'articolo analizza l'impatto che l'intervento sui sindacati, operato dal Ministero del Lavoro, produsse sul movimento dei lavoratori, in ambito rurale e cittadino, durante $i$ primi anni della dittatura civile-militare brasiliana, tra il 1964 e il 1965. Attraverso la nomina di interventores, figure scelte dalla presidenza della Repubblica o di giunte governative, lo Stato autoritario si insinuò nella struttura sindacale, obbligando le opposizioni a trovare forme di azione alternative e costringendo i dirigenti alla clandestinità, all'esilio o al carcere. Il nostro intento è discutere le ripercussioni che questi interventi ebbero sull'organizzazione del movimento dei lavoratori e sulla loro vita e valutare la portata dell'azione dello Stato contro la classe lavoratrice, obiettivo originario del golpe del 1964 e della dittatura che si instaurò successivamente.

\section{Introduzione}

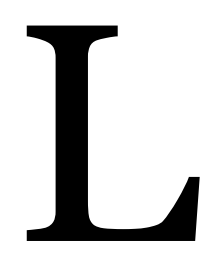

a repressione diretta contro i lavoratori durante la dittatura civile militare brasiliana è, ancora oggi, una pagina poco conosciuta della storia recente. Il movimento sindacale e la letteratura accademica sul mondo del lavoro privilegiano lo studio del periodo dell'apertura politica e della ridemocratizzazione, quando i movimenti dei lavoratori dimostravano la capacità organizzativa per la realizzazione di scioperi e interruzioni del lavoro, mobilitando una parte significativa della classe lavoratrice. Sono esemplari delle grandi mobilitazioni di questo periodo la sospensione delle attività da parte dei lavoratori della fabbrica di 
camion Saab-Scania, nell'ABC paulista ${ }^{1}$, nel 1978, e lo sciopero dei lavoratori degli engenhos, gli zuccherifici di Pernambuco, nel $1979^{2}$.

Il fenomeno conosciuto come Novo Sindicalismo, che giustappunto vide il suo inizio con lo sciopero del 1978 nell' $A B C$ paulista, fu accolto con entusiasmo dai militanti sindacali, che rivendicavano pratiche e strategie di lotta rinnovate, opponendosi così a tutta una tradizione costruita dall'egemonia comunista-trabalhista, predominante nell'organizzazione sindacale dei decenni precedenti. La proposta di avvicinarsi alla base nasceva dalla preoccupazione riguardo alla formazione politica dei lavoratori e poteva contare su un gruppo di intellettuali organici ${ }^{3}$ responsabili dell'affermazione del ruolo storico della classe lavoratrice, in forma autonoma e militante ${ }^{4}$. Come conseguenza di ciò, le esperienze di lotta dei militanti storici legati al Partito Comunista Brasiliano (PCB) e alla tradizione trabalhista furono offuscate dall'emergere del Novo Sindicalismo.

Le esperienze vissute dal Novo Sindicalismo furono accolte da una parte della letteratura accademica degli anni Ottanta con entusiasmo e simpatia. In questo contesto, tanto i vertici sindacali quanto i ricercatori (tra i quali Frederico5, Sader ${ }^{6}$ e Paranhos7) contribuirono alla costruzione di immagini e dichiarazioni che influirono direttamente sull'emergere e sul consolidarsi di nuove opportunità di lotta e suscitarono, intrinsacamente alle loro analisi e letture della congiuntura, la critica al sindicalismo burocratizzato e legato alla Stato che era stata predominante negli anni Sessanta e Settanta e aveva radici profonde nell'Era Vargas.

Come ha sottolineato Santana in occasione della ricorrenza dei quarant'anni dal 1968, avvenuta nel 2008, si sarebbe verificato da allora in poi «uno sforzo di ricerca sistematico sul periodo che va dal 1964 al 1978»8. Partendo dall'avvertimento di

\footnotetext{
${ }^{1}$ L'acronimo fa riferimento alle città industriali localizzate nellaa Regione Metropolitana di San Paolo: Santo André, São Bernardo do Campo, São Caetano do Sul e Diadema.

2 Per ulteriori dettagli su questi movimenti si vedano, rispettivamente: SANTANA, Marco Aurélio, BRAGA, Ruy, «O pêndulo oscilante: sociologia do trabalho e movimento sindical no Brasil», in Caderno CRH, 22, 56, 2/ 2009, pp. 297-309; SIGAUD, Lygia, Greve nos engenhos, Rio de Janeiro, Paz e Terra, 1980.

3 Sul concetto di intellettuale organico si veda: GRAMSCI, Antonio, Cadernos do Cárcere, Vol. 4, Rio de Janeiro, Civilização Brasileira, 2001.

4 WEFFORT, Francisco, «Os sindicatos na Política (Brasil 1955-64)», in Ensaios de Opinião, 1, 1978, pp. 2-5.

5 FREDERICO, Celso, A Esquerda e o Movimento Operário: 1964-1984, São Paulo, Novos Rumos, 1987.

${ }^{6}$ SADER, Eder, Quando Novos Personagens Entraram em Cena: experiências, falas e lutas dos trabalhadores da Grande São Paulo (1970-80), Rio de Janeiro, Paz e Terra, 1988.

7 PARANHOS, Kátia Rodrigues, Era uma vez São Bernardo. O discurso sindical dos metalúrgicos: 1971/1982, Campinas, Unicamp, 1999.

${ }^{8}$ SANTANA, Marco Aurélio, «Um sujeito ocultado: trabalhadores e regime militar no Brasil», in Revista em pauta, 12, 33, 1/2014, pp. 85-96, p. 87.
} 
Santana, questo saggio ambisce a contribuire alla conoscenza di questo periodo storico poco esplorato dalla letteratura specialistica e a gettare luce su una serie di circostanze che nel gioco della disputa per la memoria hanno perso spazio in favore del tema dei morti e dei desaparecidos nel campo della letteratura che si occupa della questione della dittatura militare brasiliana (il cui affiancamento del termine civile è già una polemica in sé). Senza dubbio, l'impatto sociale e simbolico che tante morti produssero nella società è innegabile e deve essere oggetto di tutti gli sforzi da parte dell'accademia così come dello Stato perché sia fatta piena luce su di esso. Tuttavia, studiare alcuni aspetti poco analizzati, ma che incidevano sulla vita quotidiana della classe lavoratrice, può contribuire alla comprensione dei meccanismi repressivi statali rivolti contro i lavoratori e la loro attività: gli interventi sui sindacati.

Lo studio delle interventorias ${ }^{9}$ in Brasile cerca di contribuire a colmare questa lacuna nella produzione accademica tentando, al contempo, di far cadere i tabù che riguardando il tentativo di controllo sull'ambiente sindacale come qualcosa di dinamico e dialettico. Le ingerenze sui sindacati, assieme a molti altri mezzi di repressione come le purghe, le dimissioni di massa, la persecuzione politica sui luoghi di lavoro, la disoccupazione, ebbero un impatto profondo sulla vita di centinaia di lavoratori e dei loro familiari. Queste misure, a prescindere dal fatto che non vennero considerate come gravi violazioni dei diritti, portarono gravi conseguenze per i lavoratori perseguitati e per le loro famiglie, come depressione, alcolismo, divorzi, impoverimento e accattonaggio, mancanza di autostima, di stabilità emotiva ed economica oltre ad una serie di altre questioni. Non si tratta, tuttavia, di affrontare un approfondimento delle ricerche sulle gravi violazioni perpetrate, ma di far sì che queste linee di ricerca procedano di pari passo, affinché l'una possa collaborare al chiarimento dell'altra. Vale la pena di precisare, inoltre, che la struttura del sistema repressivo, che si mostrò sin dall'inizio con l'ingerenza sui sindacati, agì nel contesto di arresti, torture, uccisioni e sparizioni.

Lo studio della repressione attuata nei confronti dei lavoratori dovrebbe quindi cercare di analizzare in modo diversificato le varie espressioni delle violazioni che toccarono questi attori, cercando così di comporre un quadro interpretativo il più ampio e complesso possibile di ciò che significò la repressione dei lavoratori durante la dittatura.

\footnotetext{
9 Il sistema delle interventorias fu uno dei principali meccanismi di centralizzazione politica e amministrativa utilizzati dall'Estado Novo di Vargas. I dirigenti statali venivano nominati da interventores, governatori dei singoli Stati federali, designati da Vargas e direttamente subordinato a lui [N.d.T.].
} 
In questo articolo presenteremo alcune interpretazioni consolidate nella letteratura specialistica, datata soprattutto agli anni Settanta e Ottanta, sottolineando alcuni limiti e contraddizioni tra gli autori. Sulla base del sondaggio condotto sul «Diário Oficial da União»10 (DOU) per gli anni 1964 e 1965, si possono rilevare gli interventi immediatamente successivi al golpe disposti dal Ministero del Lavoro e della Previdenza Sociale nei confronti dei sindacati. I dati ci consentono di misurare l'impatto del colpo di stato sulle organizzazioni associative dei lavoratori, di gettare luce sulla dimensione regionale della repressione nel paese, così come sul suo impatto sulle aree urbane e rurali.

Successivamente concentreremo l'analisi sulla documentazione dei sindacati dello Stato di Rio de Janeiro e Guanabara e su quello di San Paolo prodotti dal Ministero del Lavoro, dell'Industria e del Commercio, denominaizone dell'istituzione all' epoca del governo di varguista. Sin dal periodo di governo di Getúlio Vargas il sindacalismo in Brasile si sviluppò in stretta connessione con lo Stato. Durante il suo governo, i sindacati urbani si istituzionalizzarono e, secondo i più critici, si burocratizzarono. L'autorizzazione al riconoscimento di un sindacato dipendeva dal Ministero del Lavoro, così come l'autorizzazione alla prosecuzione di uno sciopero, l'approvazione delle elezioni sindacali, oltre a innumerevoli atti amministrativi tipici della vita sindacale. Nelle zone rurali, la sindacalizzazione dei lavoratori venne ritardata rispetto al mondo urbano, venendo raggiunta solo nel 1962, grazie alle ordinanze $\mathrm{n}^{\circ}$ 209-A/25 e 355-A del Ministero del Lavoro ${ }^{11}$.

Nei documenti esaminati possiamo trovare richieste di riconoscimento di sigle sindacali o registri delle elezioni sindacali, richieste di cambiamento di denominazione o della zona di competenza e eventuali interventi. Non tutti i documenti riportano queste informazioni in maniera completa, ma analizzandoli congiuntamente si possono dedurre alcuni modelli di azione dello Stato rivolti verso i sindacati sin dagli anni Quaranta, ma soprattutto nel contesto del Golpe del 1964 e dei primi mesi di consolidamento del regime. Anche se l'indagine nel D.O.U. mostra che le ingerenze toccarono in maniera acuta ai centri industriali qui segnalati, bisogna rilevare alcune tendenze osservate riguardao alle elezioni sindacali, alla successione dei direttori e alla stessa designazione di interventores e giunte governative da parte dell Stato brasiliano, per mezzo del Minstero del Lavoro.

\footnotetext{
${ }^{10}$ La Gazzetta Ufficiale brasiliana [N.d.T.].

${ }^{11}$ RICCI, Rudá, Terra de Ninguém: sindicalismo rural e crise de representação, Campinas, Editora da UNICAMP, 1999.
} 


\section{L'effervescenza del movimento sindacale a confronto con il frazionamento conservatore}

A differenza di ciò che una parte della letteratura ha affermato ${ }^{12}$, il periodo 1945- $^{-}$ 1964 fu marcato, all'interno del sindacalismo brasiliano, dall'azione dinamica e articolata della militanza comunista e trabalhista. Un simile contesto diede origine a un processo di democratizzazione della struttura sindacale, facendo sì che un grande numero di lavoratori potesse partecipare alla direzione delle proprie organizzazioni di rappresentanza. Questo momento, tuttavia, fu marcato da resistenze e regressi, che costituirono congiunture repressive, come quelle vissute durante i governi Dutra (194651) e Vargas (1951-54). Ricordiamo, solo per citare alcuni esempi, il lungo periodo di illegalità a cui il PCB fu costretto e le disposizioni ereditate dalla legislazione dell'Estado Novo con la Consolidação das Leis do Trabalho (CLT) ${ }^{13}$.

Nel corso della storia repubblicana brasiliana, partiti politici legati alle cause della classe lavoratrice, come il PCB dal 1922 e il PTB dal 1945, a fianco di partiti con un minor seguito, sperimentarono lunghi periodi di illegalità, venendo costretti ad agire clandestinamente. Questo arbitrio esercitato dallo Stato brasiliano si caratterizzò, in ultima istanza, come una violazione delle libertà fondamentali: la libertà di associazione, la libertà di espressione, la libertà di coscienza, diretta sistematicamente contro gli interessi della classe lavoratrice.

Secondo Lucília Delgado ${ }^{14}$, le lotte sindacali del periodo si strutturano seguendo tre assi principali: 1) allinterno della macchina statale, adeguandosi alle pratiche clientelari; 2) anche all'interno della macchina statale, utilizzando il sistema amministrativo per difendere l'autonomia sindacale e le riforme sociali; 3) agendo al di fuori dell'ambito statale e ancorandosi alle organizzazioni della società civile, incoraggiando un modello di sindacalismo che ambisse alla difesa dell'autonomia sindacale e profondamente caratterizato da un taglio riformista. Nel periodo che va dal 1955 al 1964, il sindacalismo brasiliano, guidato dall'alleanza comunista-trabalhista, appoggiò le candidature di Juscelino Kubitschek - il cui vice era João Goulart - e,

\footnotetext{
${ }^{12}$ Tra gli altri: WEFFORT, Francisco, op. cit.; BOITO Jr., Armando, Sindicalismo de Estado no Brasil, São Paulo, Hucitec, 1991.

${ }^{13}$ La Consolidação das Leis do Trabalho o Decreto-legge 5452 è una norma legislativa approvata il $1^{\circ}$ maggio 1943 - che unificò tutta la legislazione sul lavoro precedentemente esistente in Brasile. La legge, con i suoi 922 articoli, ha subito molte modifiche nel corso degli anni, ma è tuttora in vigore [N.d.T.].

14 DELGADO, Lucília de Almeida Neves, PTB: do getulismo ao reformismo (1945-1964), São Paulo, Marco Zero, 1989.
} 
successivamente, dello stesso Jango ${ }^{15}$ - in occasione della rinuncia di Jânio Quadros. La piattaforma nazionalista venne accettata da entrambi i partiti e i settori egemonici del sindacalismo brasiliano definirono, da un lato i propri spazi all'interno della struttura corporativa e, dall'altro, puntarono sulle organizzazioni intersindacali costituitesi al di fuori della logica statale, sufficientemente rilevanti in questo contesto. Per citare solo gli esempi più noti, possiamo ricordare la Comissão Permanente das Organizações Sindicais (CPOS), il Pacto de Unidade e Ação (PUA) (PUA) e Fórum Sindical de Debates (FSD) e, come punto di arrivo di questo movimento, il Comando Generale dei Lavoratori (CGT), che può essere visto come il principale portavoce delle esigenze poste da altri enti, almeno per quanto riguarda le questioni nazionali nelle aree urbane.

La vita sindacale in questo periodo era in continua crescita, al punto da essere definita da Santana come «uno dei momenti più luminosi della parabola del movimento sindacale in Brasile» ${ }^{16}$. Vediamone alcune ragioni.

In ambito sindacale si poteva osservare la proliferazione di entità intersindacali sparse per il paese, l'organizzazione di incontri sindacali e la realizzazione di innumerevoli scioperi. L'alleanza comunista-trabalhista si preoccupava di conquistare la base in misura sufficiente a occupare posti strategici allinterno della struttura corporativa, proponendo così alcuni cambiamenti al suo interno. Questo fece sì che il movimento dei lavoratori, in campagna come nelle città, potesse essere, all'inizio degli anni Sessanta, un attore politico importante, in grado di dire la propria opinione sull'evoluzione della società brasiliana. Una delle ragioni, tra le altre, del Golpe del 1964 fu interrompere questo processo di organizzazione dei lavoratori.

Nelle città, il CGT sarà una delle principali organizzazioni attraverso le quali i lavoratori potranno farsi ascoltare dalle autorità governative. Il CGT fu creato durante lo svolgimento del $\mathrm{IV}^{\circ}$ Congresso Sindacale Nazionale dei Lavoratori, tenutosi a San Paolo, nel 1962, e raccolse diverse confederazioni e organizzazioni rappresentative della classe lavoratrice ${ }^{17}$. Durante il governo Goulart, il CGT riuniva le leadership più importanti dell'epoca, a cominciare rdall'alleanza tra comunisti trabalhistas. Alla vigilia

\footnotetext{
15 Soprannome di João Goulart [N.d.T.].

16 SANTANA, Marco Aurélio, «Bravos companheiros: a aliança comunista-trabalhista no sindicalismo brasileiro (1945-1964)», in FERREIRA, Jorge, AARÃO REIS, Daniel, Nacionalismo e reformismo radical (1945-1964), Rio de Janeiro, Civilização Brasileira, 2007, pp. 235-277, p. 240.

${ }_{17}$ Come, ad esempio, la Confederação Nacional dos Trabalhadores da Indústria (CNTI), la Confederação Nacional dos Trabalhadores em Estabelecimentos de Crédito (Contec) e la Confederação Nacional dos Trabalhadores em Transportes Marítimos, Fluviais e Aéreos (CNTTMFA), la Comissão Permanente das Organizações Sindicais (CPOS), il Pacto de Unidade e Ação (PUA) e il Fórum Social de Debates (FSD).
} 
del colpo di stato del 1964, l'organismo si impegnò nel sostenere Goulart contro i golpisti, riuscendo insieme ad altre organizzazioni sociali, ad esempio, a mobilitare più di 150.000 persone il 13 marzo 1964 nella stazione Central do Brasil per il discorso del presidente Jango in difesa delle Reformas de base ${ }^{18}$.

Nelle campagne, i lavoratori si mobilitavano in misura crescente, soprattutto a partire dalla fine degli anni Cinquanta e all'inizio degli anni Sessanta. Come risultato di questo processo, sorsero diverse organizzazioni che cercavano di rappresentare questo gruppo sociale. Le Ligas Camponesas, probabilmente le più conosciute fra queste, erano associazioni di lavoratori dei campi create alla fine degli anni, inizialmente negli stati di Pernambuco e Paraíba, e che successivamente si diffusero in altre località. Vi furono anche altre importanti organizzazioni contadine, come il Movimento dos Agricultores Sem Terra (MASTER), nato nel 1960 nel Rio Grande do Sul sotto l'egida del governatore dello Stato, Leonel Brizola. Il MASTER giunse, nel marzo del 1964, fino a gestire 47 lotti in tutto lo Stato. In questo periodo fu creata anche la Confederação Nacional dos Trabalhadores na Agricultura (CONTAG), riconosciuta dal Ministero del Lavoro come rappresentante ufficiale dei lavoratori agricoli di fronte allo Stato ${ }^{19}$.

Difendendo le "reformas de base" il presidente Jango sapeva di associarsi al movimento sindacale e i lavoratori, per parte loro, avrebbero potuto appropriarsi degli spazi disponibili nella struttura statale per ottenere le loro conquiste. Tuttavia la struttura sindacale ufficiale non riuscì a essere riformata durante il suo governo $\mathrm{e}$ rimase intatta, permettendo che le singole amministrazioni statali e municipali d'orientamento conservatore, durante questo periodo e anche in quello successivo, mettessero mano alle disposizioni legislative per ingerire sui sindacati e perseguirne $\mathrm{i}$ dirigenti.

L'avanzata del movimento dei lavoratori sullo scenario politico, le minacce di sciopero e l'appoggio moderato di Goulart fecero sì che i settori conservatori, articolati attorno al binomio IPES-IBAD, sin dal 1961, e con il finanziamento e la collaborazione diretta degli Stati Uniti, diffondessero, nel bel mezzo della Guerra fredda, l'idea del pericolo dell'instaurazione di una "repubblica sindacalista" in Brasile. In questo senso René Dreifuss ha dimostrato ${ }^{20}$ come un'élite, composta da imprenditori e tecniciimprenditori, intellettuali e militari, rappresentanti di interessi finanziari

${ }^{18}$ Il comizio della Central do Brasil marcò il sostegno di buona parte delle forze di sinistra nei confronti di Goulart e provocò il successivo golpe militare [N.d.T.].

19 COMISSÃO CAMPONESA DA VERDADE, Relatório Final. Violações de direitos no campo: 1946-1988, Brasília, s.e., 2014; DE MEDEIROS, Leonilde Servolo, Trabalhadores do campo, luta pela terra e o regime civil-militar, in PINHEIRO, Milton (Org.), Ditadura: o que resta da transição, [1 $1^{\circ}$ ed.] São Paulo, Boitempo, 2014, pp. 195-230.

${ }^{20}$ DREIFUSS, René Armand, 1964: a conquista do Estado, Petrópolis, Vozes, 1981. 
multinazionali e componenti di società, si sia organizzata rapidamente intorno a un progetto politico anticomunista, basato sullo sviluppo urbano-industriale, sulla concentrazione del capitale e sull'esclusione della classe lavoratrice.

Il progetto nazional-popolare, elaborato nel corso degli anni Cinquanta e all'inizio degli anni Sessanta, decadde per effetto dell'avanzata delle forze golpiste e della conseguente deposizione del presidente João Goulart: i sindacati vennero occupati e furono nominati interventores per dirigerli, i dirigenti sindacali vennero deposti e imprigionati, i lavoratori furono perseguitati, licenziati, controllati, torturati e uccisi per mano dello Stato. I lavoratori non solo videro il loro movimento sindacale toccato all'apogeo della sua mobilitazione, ma trascorsero gli anni seguenti in un duro processo di autocritica, nel quale la ricerca dei responsabili e l'espiazione delle colpe avrebbero comportato numerosi contrasti all'interno della sinistra. Il movimento operaio non avrebbe più potuto, da allora in avanti, contare sulla struttura sindacale per organizzare la classe lavoratrice e avrebbe dovuto attendere ancora un decennio per recuperare la forza che aveva raggiunto durante il governo di Jango. Di fronte a una situazione inedita, i lavoratori si muovevano attraverso strategie alternative con l'intento di rispondere al regime militare e alle sue misure repressive e dannose per la classe, come l'imposizione di un forte compressione dei salari.

La catastrofe che rappresentò il Golpe del $1^{\circ}$ aprile del 1964 per la classe lavoratrice fu oggetto di riflessioni interne al mondo operaio e toccò il mondo accademico. Weffort ${ }^{21}$, considerando il sindacalismo populista totalmente ancorato al lavoro della sua leadership, attribuirà alla dura repressione che colpì i principali dirigenti del movimento sindacale la causa della suo smantellamento. Santana ${ }^{22}$, rivedendo questa interpretazione, nota più sfumature nella realtà dell'epoca, identificando le ragioni di questa sconfitta nella mancanza di articolazione tra i vertici e i quadri intermedi, più prossimi alla base. Fatto sta che, tra le prime vittime della repressione e della brutalità della dittatura vi saranno i dirigenti sindacali che - come vedremo - verranno perseguitati, imprigionati, o costretti alla clandestinità e all'esilio. Nei mesi che seguirono, la repressione si sarebbe estesa a tutta la classe lavoratrice e avrebbe portato in prigione un numero ancora oggi non censito di lavoratori.

Il potere politico dittatoriale che successivamente si instaurò, formato da una miriade di gruppi favorevoli a rompere con la legalità, a ridurre al silenzio e isolare politicamente le classi "pericolose", ad arginare le riforme redistributive e a consolidare

\footnotetext{
${ }^{21}$ WEFFORT, Francisco, op. cit.

${ }^{22}$ SANTANA, Marco Aurélio, Homens Partidos. Comunistas e sindicatos no Brasil, São Paulo, Boitempo, 2001.
} 
il golpe, compì uno degli sforzi iniziali nella lotta per controllare gli elementi che, potenzialmente avrebbero potuto impedire il consolidamento della dittatura. Le forze che sostenevano Goulart ed erano favorevoli alle reformas de base erano, perciò perseguitate intensamente e ciò includeva la repressione massiccia di lavoratori, sindacalisti militari lealisti e di basso grado, e politici eletti nelle file dell'opposizione. Questo fu chiaro sin dal $1^{\circ}$ aprile 1964 , come vedremo in seguito.

\section{La dittatura militare, per la classe lavoratrice, inizia il $1^{\circ}$ aprile 1964}

Il primo presidente militare, Castello Branco, farà ricorso alla legislazione sul lavoro dell'Era Vargas per fissare un controllo più rigido sul mondo del lavoro. Secondo quanto affermato da Erickson²3, l'esistenza di questo corpus normativo costituirà un meccanismo fondamentale per la destituzione di ciò che l'autore definisce l'«élite politica nazionale», molto attiva durante il governo Goulart, e per mettere le organizzazioni sindacali sotto un totale controllo statale.

La repressione militare nei confronti della classe lavoratrice ebbe inizio con l'imprigionamento o la fuga forzata dei leader sindacali e con l'assunzione del controllo sui sindacati più attivi nel paese. La resistenza operaia al Golpe guadagnò, così, lo statuto di eversione politica. Lo Stato e l'imprenditoria si garantivano il diritto di monitorare, perseguire e licenziare i propri impiegati. Imprese e Stato si associarono, internamente e al di fuori delle fabbriche, con l'obbiettivo di impedire l'organizzazione della classe lavoratrice. Nelle campagne, il rapporto tra settori dello Stato e proprietari terrieri o presunti proprietari terrieri ${ }^{24}$, la cui proprietà era dono di fabbriche e di imprese che detevano grandi proprietà terriere, mirò a neutralizzare il crescente e intenso processo di organizzazione dei lavoratori rurali, di differenti categorie, che si era sviluppato nel periodo pre-1964. Questa relazione si era già creata in precedenza, tuttavia, durante la dittatura militare, si rinsalderà ulteriormente e guadagnerà in sistematicità, organizzazione e aumento dei meccanismi repressivi.

Spesso i lavoratori vedevano le loro case violate, le loro famiglie minacciate, le sedi delle organizzazioni di categoria occupate, i loro documenti confiscati o distrutti,

23 ERICKSON, Kenneth Paul, Sindicalismo no Processo Político no Brasil, São Paulo, Brasiliense, 1979.

24 Presunti dal momento che molti di quelli che si consideravano proprietari di grandi estensioni di terre, vedevano la legittimità della loro proprietà messa in causa dai lavoratori e dalle loro organizzazioni di categoria. Questi presunti proprietari erano, generalmente, considerati dai lavoratori come grileiros (usurpatori). 
mentre moltissimi impiegati venivano licenziati, gli interventores venivano posti arbitrariamente alla direzione dei sindacati, gli scioperi erano proibiti, le dirigenze sindacali storiche venivano rimosse, azzerate, perseguitate, arrestate. Oltre agli arbitri della polizia - messi in opera con il pretesto del colpo di stato e di alcune garanzie che la CLT aveva reso possibili - l'Ato Institucional $\mathrm{n}^{\circ} 1$ permise che il governo intraprendesse una vera "caccia alle streghe", dotando lo Stato del diritto di revocare i mandati di chiunque fosse detentore di cariche elettive, oltre a privare qualunque cittadino della possibilità di assumere incarichi politici per un periodo di dieci anni. A seguito di ciò, nuove misure del governo determinarono l'accesso ai posti amministrativi e direttivi dei sindacati ufficiali, proibendo a molti sindacalisti di concorrere a cariche elettive o privandoli dei loro diritti politici o per via della loro precedente appartenenza partitica o perché avevano espresso le loro opinioni ideologiche. Il Ministero del Lavoro e le sue ramificazioni statali come le Delegacias Regionais do Trabalho (DRT), delegazioni regionali del lavoro, si incaricavano di esaminare la biografia di tutti i candidati e, all'assunzione della carica, i dirigenti eletti dovevano prestare giuramento di rispettare la Costituzione e le leggi vigenti nel paese, secondo lo stile nazionalista.

Il Golpe civile-militare toccava, in questo modo, la spina dorsale della struttura istituzionale del movimento sindacale, sopprimendo di fatto la sua capacità di mobilitazione e di pressione per la realizzazione delle proprie richieste come era stato fatto fino ad allora, ancora nei primi mesi del regime dittatoriale. Il resoconto del leader dei metalmeccanici José Ibrahim restituisce la dimensione esatta dei danni causati dalla dittatura all'organizzazione della classe lavoratrice:

Il golpe mi colpì molto, anche quando ero alle superiori, perché là c'era l'União dos Estudantes de Osasco, la UEO, e i compagni vennero arrestati, il direttorio quasi integralmente, il movimento liceale di Osasco era forte. Ed anche il sindacato subì l'intervento, infatti, assistii anche a questo: diversi compagni delle fabbriche venenro arrestati. Compresi molto bene il senso di ciò che era contro di noi. Fino al Golpe gli scioperi erano sempre stati così, basati sui picchetti, sulla forza e sull'organizzaizone dei picchetti. E i lavoratori in qualche modo speravano che vi fossero i picchetti. Se ci sono i picchetti, non entro, ho una scusante per non essere entrato. Non essendoci il picchetto, entri. Chiaramente c'erano rappresaglie, c'era gente che perdeva il lavoro, c'erano un mucchio di cose. E con il golpe e la repressione divenne impossibile organizzare i picchetti. Una cosa è mobilitare, avere il potere di mobilitazione. Se si dispone del meccanismo, se si ha l'apparato 
dello Stato in mano, così si può portare la gente nelle strade. Ma una massa che non è organizzata. In questo caso la resa dei conti, diventa una passeggiata ${ }^{25}$.

L'invasione delle sedi sindacali richiamava l'attenzione non solamente per il suo ricorrere puntualmente, ma anche perché essa rappresentava un elemento fondamentale per l'arresto e il perseguimento di diversi leader. Una delle sue conseguenze immediate fu il sequestro di una vasta documentazione che servì successivamente come materiale per l'avvio di indagini di polizia militare, Inquéritos Policiais Militares (IPM), volte ad appurare la "sovversione" in ambito sindacale. Secondo la testimonianza di Raphael Martinelli, dirigente storico della CGT, le ingerenze sui sindacati furono grandi e toccarono i settori politicamente più rivendicativi:

Vi furono interventi all'interno di tutti i sindacati. Voglio dire che tutte le aree più democratiche stavano uscendo dalla legalità che gli derivava dalla democrazia. Queste dovevano tener conto anche di tutto ciò che avrebbe potuto crear problemi, essendo democratico, alla dittatura ${ }^{26}$.

Negli Stati in cui il movimento sindacale era politicamente più organizzato come São Paulo, Rio de Janeiro, Pernambuco, Bahia, Minas Gerais e Rio Grande do Sul, si verificarono, nel periodo immediatamente successivo al golpe, arresti preventivi dei leader sindacali, impostando così un quadro di repressione preventiva o immediata di ogni forma di organizzazione e manifestazione collettiva. Un'esemplificazione di questo progetto di Stato autoritario è rilevabile nella sospensione decennale dei diritti sindacali di Clodesmidt Riani, presidente della confederazione nazionale dei lavoratori delloindustria, Confederação Nacional dos Trabalhadores da Indústria (CNTI) e della CGT. Il governo militare cercò, come strategia ideologica, di legare la CGT al "comunismo internazionale". Il Ministro del Lavoro Arnaldo Sussekind comparve in televisione a pochi giorni dal Golpe per accusare Raphael Martinelli di essere "agente di Mosca”, facendo riferimento al discorso da lui tenuto in qualità di capo della delegazione brasiliana al Congresso dei Sindacalisti Sovietici del 1963.

25 Tratto dal documentario 1964 um golpe contra o Brasil, di Alípio Freire. FREIRE, Alípio, 1964 um golpe contra o Brasil, Núcleo de Preservação da Memória Política - TVT, Brasil, 2013, 146'. Disponibile anche all'URL: < https://www.youtube.com/watch?v=R64NQsn2rYc > [consultato il 28 ottobre 2014].

${ }^{26}$ Projeto Memória da Oposição Sindical Metalúrgica de São Paulo. URL:

< https://www.youtube.com/watch?v=kYo77rMwjwk > [consultato il 28 ottobre 2014]. 
Nelle campagne la storia si ripeteva. Tra i principali dirigenti della CONTAG c'era Lyndolpho Silva, Presidente della confederazione. Anche a lui, nel 1965, vennero revocati i diritti politici per dieci anni e rimase in clandestinità, con il rischio di essere arrestato, fino al 1973 quando decise di andare in esilio in Europa, stabilendosi a Praga, dove rimase sino al ritorno in Brasile, nel 1979, beneficiando della Legge di amnistia. Altri dirigenti, che avevano assunto la direzione delle federazioni statali, come ad esempio Manoel Gonçalo Ferreira di quella di Pernambuco, videro revocati i loro diritti politici e, perseguitati, furono obbligati a restare per molti anni in clandestinità ${ }^{27}$.

Il sindacato dei metalmeccanici di Rio de Janeiro, riferimento per la lotta dei lavoratori a livello nazionale, divenne immediatamente, allo stesso modo, obiettivo delle forze golpiste. Il giorno del colpo di stato la sede dell'orgazzazione venne occupata dalle forze di polizia, che ne demolirono praticamente tutto l'edificio, distruggendo le sezioni, le stanze, la mobilia e la documentazione. Nei giorni successivi il Ministro del Lavoro Arnaldo Sussekind emanò un'ordinanza intervenendo in maniera formale nel sindacato nominando una Giunta governativa, composta da dirigenti conservatori dell'organizzazione che avevano già fatto parte dei vertici negli anni Cinquanta, quando questa era guidata da comunisti e trabalhisti.

Secondo Santana ${ }^{28}$, il 20 aprile del 1964 circolavano tra i metalmeccanici due documenti, controfirmati dalla Giunta governativa. Questi documenti informavanod el fatto che la Giunta era in contatto con il Ministero del Lavoro e che aveva proposto l'ingresso di tre sindacalisti per integrarla, dimostrando - anche a livello di direzione sindacale - la collaborazione del settore operaio, che grazie al golpe avrebbe avuto l'occasione di sconfiggere l'egemonia comunista-trabalhista che era stata sino a quel momento alla guida dell'organizzazione. Obbedendo all'ideologia golpista, il documento chiedeva ai lavoratori di appoggiare la giunta e accusava la direzione precedente di agitazione politica. Il loro obiettivo era mantenere, fedelmente alla loro filosofia, l'organizzazione «al di sopra della lotta politico-partitica»29.

La Giunta appena insediata fu allora responsabile di indicare una lista di membri dell'antica direzione, che - ad eccezione del metalmeccanico Heraclides Santos dovevano essere ritenuti responsabili per il clima di insicurezza venutosi a creare. Così,

27 CARNEIRO, Ana, CIOCCARI, Marta, Retrato da repressão política no campo - Brasil 1962-

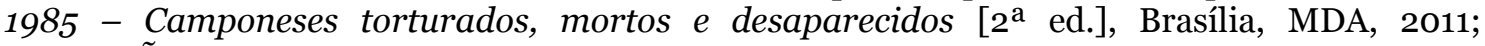
COMISSÃO CAMPONESA DA VERDADE, Relatório Final. Violações de direitos no campo: 1946-1988, Brasília, s.e., 2014.

28 SANTANA, Marco Aurélio, "Os sindicatos e o golpe de Estado de 1964: a experiência dos metalúrgicos do Rio de Janeiro", in Revista Perseu: história, memória e política : Dossiê Organização dos Trabalhadores: das sociedades de resistência ao sindicalismo contemporâneo, 7, 10, 2013, pp. 33-60.

29 Ibidem, p. 49. 
per mezzo di un dispaccio del direttore-generale del Dipartimento Nazionale del Lavoro, Departamento Nacional do Trabalho (DNT), ai nomi indicati dalla Giunta fu impedito di svolgere incarichi in organizzazioni sindacali per motivi politici, anche allo stesso che era stato risparmiato dai membri della Giunta. La relazione della Giunta indicava che nelle procedure del Ministero del Lavoro per la determinazione dei nomi che avrebbero fatto parte della nuova giunta, la Confederação Nacional dos Círculos Operários, nella persona del suo assistente ecclesiastico, Padre Veloso, svolse un ruolo importante ottenendo incarichi dirigenziali nel sindacato, come quello concesso al circulista Giovanni Américo Maranhão30. Ad aggravare ancor più la situazione dei comunisti e dei trabalhistas perseguitati dalla Giunta, venne varato l'IPM n. 709, che aveva come scopo l'individuazione delle attività comuniste nel paese e che faceva capo direttamente al Sindacato dei Metalmeccanici di Rio de Janeiro, e che portò all'arresto di diversi militanti. Per quanto riguarda i membri della direzione destituiti dal loro incarico, Santana registra che, per diversi motivi, nessuno di loro poté riprendere la propria militanza sindacale, neanche alla conclusione del periodo di governo militare, segno dell'impatto politico e sociale che queste violazioni ebbero sulla vita di diversi sindacalisti ${ }^{1}$.

Anche i Círculos Operários rappresentarono una forza importante nel periodo postgolpe, dal momento che molti dei loro quadri vennero nominati interventores nell'ambito del sindacalismo agrario. L'interventor nominato dal Ministero del Lavoro per la presidenza della CONTAG, José Rotta, proveniva da questa organizzazione, oltre ad essere un dirigente sindacale paulista. Lo stesso avvenne con le federazioni di São Paolo e di Rio de Janeiro. Si deve evidenziare come molti dei quadri nominati dal governo militare fossero stati militanti sindacali nelle campagne. È il caso, ad esempio, di Acácio Fernandes dos Santos, la cui attività si svolgeva principalmente nell'area di San Gonçalo, nella regione metropolitana dello Stado di Rio de Janeiro. Acácio divenne presidente della Federazione dei Lavoratori agricoli dello Stato di Rio de Janeiro successivamente al Golpe ${ }^{32}$. Tuttavia, come sottolinea Medeiros ${ }^{33}$, non dobbiamo

${ }^{30}$ Il circulismo fu un movimento legato alla Chiesa cattolica Católica ed ebbe la sua esperienzapilota tra gli operai. I Círculos Operários cercavano di porre rimedio alle condizioni di povertà in cui vivevano i lavoratori e, allo stesso tempo, rifiutando qualsiasi possibilità di mutamento sociale attraverso la rottura, trasferivano tutti i problemi del mondo del lavoro alla sfera della cooperazione. Questo movimento si avvicinò ai militari al momento del golpe e, da allora, realizzò con loro una alleanza che permise ai Círculos di assumere la direzione di alcune organizzazioni sindacali, mantenendole in attività.

${ }^{31}$ SANTANA, Marco Aurélio, "Os sindicatos e o golpe de Estado de 1964: a experiência dos metalúrgicos do Rio de Janeiro», cit.

$3^{2}$ TEIXEIRA DOS SANTOS, Marcos Antonio, Os trabalhadores rurais e as lutas no campo na Baixada: 1964-1979, Rio de Janeiro, Unirio, 2009. 
semplificare l'interpretazione dell'attività di ingerenza di questi settori della Chiesa. Secondo l'autrice, l'azione di molti di questi era:

[...] profondamente intrisa della necessità di incentivare l'organizzazione dei lavoratori agricoli e portatrice di una concezione dei diritti che, se da un lato si allontanava dai principi comunisti, dall'altro faceva sì che venisse difesa l'urgenza di combattere le differenti forme di oppressione che caratterizzavano la forma tradizionale di sfruttamento vigente nelle fazende e negli engenhos ${ }^{34}$.

Gli esempi qui citati sono esplicativi di uno degli argomenti che questo lavoro cerca di rafforzare: le ingerenze sui sindacati generalmente non erano azioni isolate del regime militare che si era costituito, ma parte di un più vasto insieme di violazioni indirizzate contro i lavoratori.

Le ingerenze sui sindacati non furono un'esclusiva che toccò i sindacati maggiori e meglio organizzati, ma si caratterizzarono come una pratica persecutoria ricorrente ed efficace nel periodo immediatamente successivo al golpe, nella misura in cui fornivano agli agenti dello Stato autoritario le informazioni più affidabili e sistematiche sui militanti. Tra l'aprile e il maggio del 1964, secondo una rilevazione statistica dei «Diários Oficiais da União», il Ministero del Lavoro nominò 235 interventores e intervenne direttamente in sette delle dieci confederazioni, tra cui la CGT, che era la stata la spina dorsale del movimento sindacale sino ad allora, e la CONTAG che, come accenanto più sopra, era di recente creazione. Spettava agli interventores investigare, identificare e denunciare il passato dei leader sindacali più noti, suggellando così il futuro dei dirigenti che avrebbero progettato la resistenza e la lotta come mezzo per ottenere le loro richieste. Una delle conseguenze immediate per la vita sindacale fu lo svuotamento del sindacato, come riporta il grafico Nilton Pedrosa: «Soprattutto quando [la sede del sindacato] era qui nella [Avenida] Presidente Vargas, poca gente ci andava perché aveva paura. Perché c'era gente, c'erano spioni. La gente non ne era direttamente a conoscenza, ma aveva ben inteso» 35 .

Il sistema legale che garantì al Ministero del Lavoro il diritto di intervenire sugli enti sindacali non fu una novità del regime militare, ma era garantito sin dal 1943 dalla CLT. Tuttavia, senza dubbio durante il governo Castello Branco lo Stato brasiliano,

33 DE MEDEIROS, Leonilde Servolo, Trabalhadores do campo, luta pela terra e o regime civilmilitar, cit.

34 Ibidem, p. 201.

35 Testimonianza di Nilton Pedrosa e Luiz Batista Bruno di fronte alla Comissão Nacional da Verdade (CNV) do Brasil e alla Comissão Estadual da Verdade do Rio (CEV-Rio), 5 agosto 2014. 
attraverso il ministero di cui sopra, fece uso in modo repressivo e sistematico di questo dispositivo autoritario. Durante il regime militare, gli interventi in ambito sindacale poterono contare in misura sempre crescente su meccanismi legali che aggiornavano il tema, permettendo così il superamento della CLT. Durante la dittatura militare brasiliana, le ingerenze sui sindacati guadagnarono uno status di legalità nella misura in cui altre pratiche repressive rivolte contro la classe lavoratrice andavano delineandosi sullo scenario politico (creazione del Fundo de Garantia do Tempo de Serviço - FGTS e dell'Instituto Nacional do Seguro Social - INSS, politica di compressione salariale, licenziamenti di massa, legislazione anti-sciopero, etc.). Secondo Celso Frederico ${ }^{36}$, le relazioni tra lo Stato e il movimento sindacale durante la dittatura militare possono essere considerate come il momento in cui la questione sociale divenne oggetto di interesse per la sicurezza nazionale.

Il 21 gennaio 1965, il Ministro del Lavoro Arnaldo Sussekind pubblicò l'Ordinanza n. 40, conosciuta tra i lavoratori come l'“AI-5 del movimento sindacale”. Come spiega Santana: «istruiva gli interventores affinché avviassero procedimenti contro i membri delle direzioni deposte dal golpe, per presupposte irregolarità, impedendo loro, per effetto di quanto veniva disposto nell'ordinanza, di rientrare nel sindacato tramite elezione»37. Veniva espressa chiaramente l'intenzione del governo di controllare i sindacati attraverso la scelta dei suoi dirigenti.

Gli interventores si inserirono, indirettamente, nella struttura del Serviço Nacional de Informações (SNI), integrando così le propaggini dello Stato all'interno del movimento sindacale. Luigi Negro definisce il ruolo dell'interventor in base a tre funzioni principali:

[...] i sindacalisti di fiducia del nuovo regime dovevano svolgere tre funzioni principali. Si doveva, inizialmente, dare continuità alla vigilanza, facendo del sindacato un luogo di identificazione degli attivisti ancora in attività. La loro seconda funzione consisteva nello spostare l'attività dei sindacati dal campo della rivendicazione di migliori condizioni di lavoro all'assistenzialismo. L'ipertrofia delle funzioni assistenziali e l'orientamento politico degli interventores svincolavano i sindacati dalle questioni specifiche dei luoghi di lavoro alla situazione generale delle categorie che rappresentavano. Infine gli interventores dovevano contenere tanto le opposizioni sindacali quanto le mobilitazioni che mettevano in discussione la politica della dittatura militare e l'autorità

${ }^{36}$ FREDERICO, Celso, A Esquerda e o Movimento Operário - 1964-1984, São Paulo, Novos Rumos, 1987.

37 SANTANA, Marco Aurélio, Homens Partidos. Comunistas e sindicatos no Brasil. São Paulo, Boitempo, 2001, p. 151 
dell'imprenditoria, neutralizzandone o scoraggiondone le azioni a partire dai luoghi di lavoro ${ }^{38}$.

Emerge, in questo caso, il ruolo assunto dai Dipartimenti Nazionali del Lavoro (i già citati DRT), assieme al Ministero del Lavoro e ai Dipartimenti per l'Ordine Pubblico e Sociale, Departamentos de Ordem Política e Social (DOPSs), nel controllo sindacale. All'interno di questa logica toccò ai DRT seguire le tappe delle elezioni sindacali, con l'appoggio molto stretto degli apparati repressivi dello Stato. L'obiettivo di depoliticizzare il movimento sindacale può essere esemplificato con ciò che João Guilherme Vargas Neto ${ }^{39}$ ha chiamato "voto sindacale colorado": le liste che si costituivano, invece di nomi che potessero ricondurle a questioni politiche, avevano colori per contraddistinguerle (azzurro, verde, giallo, arancione e rosso). Ovviamente, la lista rossa, benché esistente, non era "raccomandata" dalla Delegazione. Il peso repressivo di questo controllo fu, inoltre, accompagnato dall'utilizzo di "liste nere", dalla corruzione, dalle frodi e dal furto di voti, trasformando così le elzioni sindacali in una vera odissea, quasi sempre con un esito sfavorevole alle liste che si opponevano agli interventores.

A differenza di altri segmenti sociali perseguitati dal regime, la dittatura, per i lavoratori, iniziò effettivamente il $1^{\circ}$ aprile del 1964. L'intenzione era quella di destrutturare il vigoroso movimento dei lavoratori che si era costituito e impedire qualsiasi possibilità di resistenza al Golpe. Per questa ragione l'apparato dello Stato fece ricorso a diversi meccanismi di repressione e controllo. Questa azione portata avanti contro la classe lavoratrice ebbe luogo, come abbiamo visto, con modalità differenti, avendo come costante la strategia di intimidazione, la divisione politica dei militanti, la burocratizzazione delle organizzazioni di rappresentanza e colpendo i diritti fondamentali, come quello di espressione e di libera associazione.

\section{Il controllo statale sui sindacati attraverso le intervenções}

Lo studio delle intervenções, gli interventi statali in ambito sindacale, rafforza la tesi - esposta nel capitolo Violações de direitos humanos dos trabalhadores del

${ }^{38}$ NEGRO, Antonio Luigi, Nas Origens do 'Novo Sindicalismo': O Maio de 59, 68 e 78 na Indústria Automobilística, in RODRIGUES, Iram Jácome (Org.), O Novo Sindicalismo. Vinte Anos Depois, Petrópolis, Vozes, 1999, p. 17.

39 VARGAS NETO, João Guilherme, «O voto sindical colorido», in ZéDirceu, 14 gennaio 2014, URL: < http://www.zedirceu.com.br/o-voto-sindical-colorido/ > [consultato l'11 novembre 2015]. 
rapporto finale della Comissão Nacional da Verdade do Brasil - secondo cui l'allestimento del golpe dell'aprile 1964 aveva come obiettivo principale la classe lavoratrice. La frenesia e la combattività del movimento sindacale e la posizione conciliatrice del governo Goulart minacciavano gli interessi economici che desideravano rendersi protagonisti impossessandosi dello Stato, in collaborazione con le Forze Armate.

Le ingerenze statali sul mondo sindacale comprovano, in una certa misura, come il golpe fosse "di classe". Gli interventores svolsero un ruolo centrale nell'immediato post-golpe, nella misura in cui sostituirono i membri dei direttivi allontanati, scomparsi o catturati - nel qual caso portavano ad un'acefalia dell'organizzazione - provocando un rinnovamento nei quadri sindacali, ma in ogni modo una maggiore ingerenza dello Stato sulle organizzazioni. I membri delle giunte governative o gli interventores erano designati dal Ministero del Lavoro o, in alcuni casi, dal delegato regionale del lavoro. I loro mandati variavano in durata: potevano essere di 15 giorni per regolarizzare una situazione specifica, per esempio procedere ad analizzare i conti del sindacato, o per un mese, affinché si potessero organizzare nuove elezioni. Le intervenções erano considerate dallo Stato come straordinarie, giustificate solamente da una sitauzione imprevedibile e che doveva essere utile ad una regolarizzazione delle attività sindacali.

Come ha affermato Mattos ${ }^{40}$, tra 1964 e 1967, gli interventores erano dirigenti in gran parte legati, come abbiamo visto, ai Círculos Operários Católicos e al sindacalismo statunitense che avevano perso, negli anni di Goulart, la loro egemonia a vantaggio del PCB e del PTB. Si presentarono così come il punto d'appoggio dei primi governi militari e perseguitarono i loro avversari, tacciati di essere pericolosi sovversivi. Nella CONTAG e nelle federazioni di lavoratori rurali di San Paolo e di Rio de Janeiro, la giunta governativa era composta da sindacalisti legati ai circoli. Nel Nordeste, nelle federazioni di Pernambuco e del Rio Grande do Norte, le persone scelte provenivano dal Serviço de Orientação Rural, legati al vescovato, come nel caso del Serviço de Orientação Rural de Pernambuco (Sorpe) ${ }^{41}$.

Più di recente il tema delle intervenções ha fatto la sua comparsa nelle tesi accademiche, anche se, nella maggior parte dei casi in forma sufficientemente tangenziale. In occasione del cinquantenario dal Golpe del 1964 si è potuto assistere ad un boom editoriale di libri e periodici che, sollecitati dalla data, hanno colto l'occasione per rivedere temi molto dibattuti e aprire nuovi fronti nella ricerca. La data ha

${ }^{40}$ MATTOS, Marcelo Badaró, Do Golpe à nova transição democrática, in ID., Trabalhadores e Sindicatos no Brasil, São Paulo, Expressão popular, 2009, pp. 101-115.

${ }^{41}$ DE MEDEIROS, Leonilde Servolo, Trabalhadores do campo, luta pela terra e o regime civilmilitar, cit. 
acquistato ancora più rilevanza in un contesto in cui erano in corso sia i lavori della Comissão Nacional da Verdade brasiliana, sia quelli di una serie di commissioni per la verità di livello regionale e settoriale e una vasta documentazione prodotta dagli organi di repressione era stata da poco inventariata e resa disponibile al pubblico dall'Archivio Nazionale.

Generalmente in questi lavori42 il tema delle intervenções appare sempre come un elemento secondario. Infatti, come abbiamo mostrato, gli studi sulla repressione dei lavoratori hanno giocato un ruolo secondario tra gli oggetti di studio presi in esame dalla produzione accademica sul tema della dittatura brasiliana. Le intervenções appaiono così come un capitolo della repressione dei lavoratori, quasi sempre inseriti in un quadro di aspra repressione del sindacato. Questi lavori recenti si sono limitati a riportare i dati degli studi realizzati negli anni Settanta e Ottanta relativamente alle intervenções, il che denuncia un evidente abbandono del tema da parte dell'accademia, per lo meno negli ultimi 25 anni.

Degno di nota, tuttavia, è il recente lavoro realizzato da Nagasava 43 nel quale l'autrice dedica un capitolo al tema. Lo studio mira a sviluppare una riflessione più ampia sui meccanismi di controllo in ambito sindacale portati avanti dal Ministero del Lavoro, apportando come giustificazione ideologica ciò che sarà chiamato, come dimostra l'autrice, novo trabalhismo.

Questo saggio, a prescindere dal fatto che si serve soprattutto di dati statistici, almeno in questa prima fase di ricerca, ambisce a gettare luce tanto sull'impatto delle intervenções nell'organizzazione dei lavoratori, soprattutto, nell'articulazione comunista-trabalhista, quanto sui gruppi che emersero in questa nuova situazione, per effetto della ristrutturazione della struttura sindacale.

Tuttavia, come vedremo, non di rado si verificarono casi in cui i dirigenti sindacali rimasero in carica prima e dopo il Golpe, quando la maggior parte dei sindacati erano amministrati da interventores. In altri casi constatiamo che membri delle giunte governative vennero eletti presidenti del sindacato dopo la regolarizzazione delle loro attività. Quando prendiamo in considerazione questa pluralità di situazioni, emerge la

42 Tra gli altri: REIS FILHO, Daniel Aarão, Democracia e Ditadura no Brasil: do golpe de 1964 à Constituição de 1988, Rio de Janeiro, Zahar, 2014; NAPOLITANO, Marcos, 1964: História do Regime Militar Brasileiro, São Paulo, Editora Contexto, 2014; MATTOS, Marcelo Badaró, VEGA, Rubén (Org.), Trabalhadores e ditaduras: Brasil, Espanha e Portugal, Rio de Janeiro, Consequência, 2014.

43 NAGASAWA, , Heliene Chaves, "O sindicato que a ditadura queria": o Ministério do Trabalho no governo Castelo Branco (1964-1967), Rio de Janeiro, Dissertação de Mestrado Acadêmico em História, Política e Bens Culturais apresentada ao Centro de Pesquisa e Documentação de História Contemporânea do Brasil - CPDOC, 2015. 
necessità di rivolgere uno sguardo attento al funzionamento della vita sindacale e ai meccanismi di ingerenza dello Stato su questa.

Le informazioni che troviamo sul tema nella letteratura specializzata e sui periodici della classe lavoratrice sono isolate e inesatte. Rivisitiamo dunque la letteratura che si è occupata della repressione dei lavoratori durante la dittatura con l'intento di dare risposta a questioni aperte e a individuare eventuali imprecisioni nei dati. Come vedremo in seguito si riscontra una certa ambiguità nei numeri e una concentrazione a livello regionale che impedisce una visione d'insieme delle conseguenze e del senso sugli ingerenze nel sindacato nel post-Golpe (la maggior parte delle rilevazioni sulle intervenções si concentrano nella regione del Sudeste, specialmente nello Stato di São Paulo).

Nonostante vi sia differenza tra le rilevazioni delle intervenções raccolte dai ricercatori e quelle del movimento sindacale è certo che le intervenções furono abbondanti e toccarono più fortemente i grandi sindacati (con più di 5.000 associati), oppure quelle più poderose e politicamente più attive.

Qui incontriamo la prima imprecisione presente nella letteratura: il numero dei sindacati che subirono le ingerenze statali. Come afferma Nagasava44, il numero comunemente adottato, tanto dai ricercatori quanto dai sindacalisti, è quello di 433 intervenções occorse nell'immediato periodo successivo al Golpe, tra il 1964 e il 1965 , secondo le rilevazioni fatte da Figueiredo 45 dai D.O.U. In altre opere, tuttavia, i numeri tendono a salire: 537 intervenções, secondo Erickson ${ }^{46} ; 761$ intervenções, secondo Heloísa Martins47; 831 intervenções, secondo Moreira Alves48 (1985), sempre per il biennio 1964-1965. La polemica si estende ai periodi successivi, rendendo necessaria la revisione e la verifica dei dati utilizzati sino ad ora.

Questa prima tappa di ricerca, relativa al biennio 1964-1965, ha permesso in primo luogo di dare misurare l'impatto del Golpe sulle organizzazioni sindacali, identificando quali e quante di loro subirono interventi statali. Abbiamo deciso di concentrarci su questi due anni dal momento che, oltre ad essere indicati dalla letteratura come il lasso

44 NAGASAWA, Heliene Chaves, "O sindicato que a ditadura queria": o Ministério do Trabalho no governo Castelo Branco (1964-1967), Rio de Janeiro, Dissertação de Mestrado Acadêmico em História, Política e Bens Culturais apresentada ao Centro de Pesquisa e Documentação de História Contemporânea do Brasil - CPDOC, 2015.

45 FIGUEIREDO, Marcus, KLEIN, Lúcia, Legitimidade e Coação no Brasil pós-64, Rio de Janeiro, Forense Universitária, 1978.

46 ERICKSON, Kenneth Paul, Sindicalismo no Processo Político no Brasil, São Paulo, Brasiliense, 1979.

47 TEIXEIRA De SOUZA MARTINS, Heloísa Helena, O Estado e a Burocratização do Sindicato no Brasil, São Paulo, Hucitec, 1979.

${ }^{48}$ ALVES, Maria Helena Moreira, Estado e Oposição no Brasil (1964-1984), Petrópolis, Vozes, 1984. 
di tempo con la massima concentrazione di intervenções, si caratterizza come un periodo ottimale per verificare rotture e continuità nella politica sindacale e nella relazione fra Stato e sindacati. In secondo luogo, lo studio delle intervenções nell'immediato post-Golpe ha contribuito a comprovare la tesi secondo cui la repressione è iniziata, per la classe lavoratrice, il $1^{\circ}$ aprile 1964. In terzo luogo si può osservare come le intervenções siano state, in molte occasioni, accompagnate da altre violazioni nei confronti dei diritti dei lavoratori, come l'imprigionamento dei leaders sindacali, la spoliazione del patrimonio fisico e materiale delle organizzazioni sindacali, la confisca o la distruzione di documenti, il licenziamento, la revoca di mandati sindacali e parlamentari, ...

\subsection{L’analisi dei «Diários Oficiais da União» (D.O.U.)}

Di fronte a una tale incertezza riguardo alle ingerenze sui sindacati avvenute subito dopo il Golpe, questo lavoro propone di realizzare una nuova rilevazione a partire dal D.O.U. per gli anni 1964 e 1965, in modo da identificare il numero esatto delle intervenções e venire a capo di questa controversia storiografica. Sono stati consultati i numeri relativi a 22 mesi di pubblicazioni del D.O.U., dal marzo 1964 al dicembre 1965. Molti decreti relativi agli interventi statali messi in atto nel 1964 furono pubblicati sul D.O.U. solamente molti mesi dopo.

Nel settore del Ministero del Lavoro e della Previdenza Sociale furono registrate dal D.O.U. 235 intervenções. La maggior parte di queste avvenne nel corso dell'anno 1964, soprattutto nei primi mesi dopo il Golpe, il che corrobora la tesi secondo cui i lavoratori sarebbero stati il principale obiettivo delle forze militari associatesi con la classe imprenditoriale. Queste si concentrarono soprattutto in ambito urbano, nei sindacati attivi in ambito nazionale e in particolare in quelli degli Stati di São Paulo, di Rio de Janeiro e Guanabara.

L'articolo 528 della CLT funzionò come giustificazione legale alle intervenções. L'articolo recitava chiaramente che:

[...] verificandosi dissidi o controversie che inficiano il funzionamento del sindacato, il ministro del Lavoro, dell'Industria e del Commercio potrà intervenire in queste attraverso delegati dotati di poteri per l'amministrazione delle 
associazioni e mettere in opera o proporre le misure necessarie per normalizzarne il funzionamento49.

È opportuno registrare che questo articolo ottenne una nuova formulazione con il Decreto Legge n. 3, del 27 gennaio 1966, includendo, assieme alle circostanze che disturbavano il funzionamento delle organizzazioni sindacali, anche «motivi rilevanti di sicurezza nazionale».

Le intervenções confermavano, dunque, una chiara ingerenza dello Stato sulle organizzazioni sindacali, attraverso il Ministero del Lavoro. I sindacati furono utilizzati dal regime militare come canale prioritario per il controllo della classe lavoratrice. Lungi dall'opporsi alla loro esistenza, il ministro del Lavoro Arnaldo Sussekind (19641965), attraverso la nomina di interventores o di giunte governative, cercò di stabilire un controllo diretto sui sindacati, sia mediante l'allontanamento dei dirigenti considerati sovversivi, sia sostenendo la decapitazione delle organizzazioni con la sparizione di molti dirigenti sindacali, imprigionati o obbligati alla clandestinità. In un innumerevole numero di ordinanze ministeriali troviamo questa formulazione: «In considerazione del fatto che diversi dirigenti del Sindacato [...] sono irreperibili, senza che si possa comprovare l'esistenza di un atto esplicito di rinuncia o di una atto regolare che li destituisca dai loro incarichi»50.

Questa terminologia finisce per essere generalizzata, da Frederico, facendo riferimento all'acefalia del movimento operaio come ad un tuttuno:

Il periodo compreso tra il golpe del 1964 e la metà del 1966 è marcato dalla compatta azione repressiva del governo, dallo smantellamento e dalla decapitazione dei vertici del movimento operaio. La reazione dei lavoratori si limitò a pochi scioperi isolati e ai tentativi di riconquista dei sindacati toccati dagli interventi statali51.

Successivamente ai dirigenti destituiti dopo il 1964 venne impedito di ritornare alla direzione delle organizzazioni attraverso l'elezione, secondo quanto stabilito dall'Ordinanza n. 40, del 21 gennaio 1965, riportata più sopra.

49 Consolidação das Leis do Trabalho, art. 528. Disponibile all'URL: < http://www.planalto.gov.br/ccivil_03/decreto-lei/Del5452.htm > [Consultato il 9 settembre 2015].

50 Si veda, ad esempio, l'Ordinanza n. 274, del 28/04/1964, del Sindacato degli Operai dei cantieri navali di Rio de Janeiro, pubblicata nei D.O.U. del 04 maggio 1964, Sezione 1, p. 17.

${ }^{51}$ FREDERICO, Celso, op. cit., p. 21. 
Il Ministero del Lavoro promosse, attraverso le intervenções articolate assieme ad altri mezzi di repressione e a modifiche della legislazione del lavoro vigente, una vera e propria burocratizzazione sindacale, rendendo obbligatorio l'invio di un rapporto sulle attività annuali, una serie di documenti e di autorizzazioni necessarie per il pieno funzionamento dell'associazione: il tutto per un maggior controllo da parte dello Stato. Un tale controllo richiedeva la garanzia che ai precedenti dirigenti venisse impedito di riprendere le attività in campo sindacale, quantomeno in via ufficiale e, allo stesso tempo, ci si preoccupava di assicurare "la normalizzazione della vita sindacale", attraverso l'elezione di lavoratori di fiducia. In circa il 90\% dei casi analizzati, gli interventi statali avevano come principale obiettivo la realizzazione delle elezioni per la normalizzazione della vita dell'organizzazione. Il termine fissato poteva variare tra $15 \mathrm{e}$ 90 giorni. Era comune la proproga di queste scadenze, quando l'elezione era avvenuta in circostanze turbolente o veniva contestato dagli ex leaders.

Oltre alla già citata decapitazione dei vertici dell'organizzazione, il motivo più frequentemente ricorrente dell'ingerenza statale era dato dall'interdizione della sede del sindacato o della federazione ${ }^{52} \mathrm{o}$ dall'accusa di attività sospette da parte del direttivo eletto53.

Le intervenções avvenivano con due modalità distinte: la più comune si verificava per mezzo della nomina di una giunta governativa, composta da un presidente, un segretario e un tesoriero; la seconda attraverso la designazione di un Delegato Sindacale che potesse svolgere il ruolo di interventor fino alla normalizzazione delle aattività dell'organizzazione. Nel primo caso, gli agenti nominati erano appartenenti alla prima categoria; nel secondo caso si trattava di attori esterni all'ambito sindacale.

\subsection{L'analisi delle schede sui sindacati}

In modo complementare all'analisi dei D.O.U., sono state esaminate due raccolte di documenti rinvenute nel 2014 presso il Ministero del Lavoro e dell'Impiego, il

$5^{2}$ «[...] in considerazione del fatto che le autorità di polizia e militari, a seguito di perquisizioni effettuate nella sede del Sindacato [...] per appurare la participazione dei propri dirigenti ad attività sovversive, lo interdicono", così come appare, ad esempio, nell'Ordinanza n. 275, del 28/04/1964, Sindacato degli impiegati nelle attività alberghiere e affini di Rio de Janeiro, D.O.U. del 4 maggio 1964, Sezione 1, p. 18.

53 «[...] l'azione della sua amministrazione ha costituito una causa determinante di questa situazione», secondo l'Ordinanza n. 386, del 15/05/1964, del Sindacato dei ferrovieri di Vitória, D.O.U. del 18 maggio 1964, Sezione 1, p. 16. 
Ministério do Trabalho e Emprego (MTE) ${ }^{54}$. Si tratta di schede sui sindacati elaborate dal Ministero del Lavoro, dell'Industria e del Commercio - denominazione del ministero durante il governo Vargas - relativi allo Stato di Rio de Janeiro e Guanabara e allo Stato di São Paulo. I documenti coprono gli anni dagli anni Quaranta agli anni Settanta e registrano, in maniera sintetica, le principali attività amministrative esercitate dall'organizzazione sindacale. Non sappiamo per quale raggione i documenti rispondano a questa logica di classificazione cronologica, ma ci sono assolutamente utili per gli intenti di questa ricerca. Tra questa documentazione possiamo trovare di tutto: dalla richiesta di riconoscimento sindacale al ministero di competenza, fino alle date delle elezioni sindacali, la composizione del loro direttivo, il registro degli eventuali interventi da parte dello Stato e delle lotte interne per la direzione dell'organizzazione. Il materiale offre, perciò, la possibilità di verificare il funzionamento dei sindacati non solamente quando svolgevano le loro attività in forma anormale (soggetti a un intervento statale), ma anche durante $\mathrm{i}$ periodi di funzionamento regolare.

A partire dall'analisi di questa documentazione, è stato possibile misurare la portata del Golpe per la vita sindacale negli anni iniziali della dittatura nei sindacati dei due principali centir urbani del paese: Rio de Janeiro e San Paolo. Anche se non possiamo superare la concetrazione di analisi per l'asse Rio-San Paolo, i dati comprovano, una volta di più, la forte repressione rivolta contro i lavoratori e l'intento da parte dello Stato, attraverso il Ministero del Lavoro, di ottenere il controllo sulle organizzazioni sindacali dei lavoratori e ridefinire il ruolo del sindacato come fornitore di servizi e benefici, rafforzando la sua funzione assistenziale.

In totale, sono state consultate 133 schede di organizzazioni sindacali dello Stato di Rio de Janeiro e Guanabara e 331 riferite a organizzazioni dello Stato di São Paulo, come si può osservare nel grafico (Fig. 1.). Come si può osservarequesta gran mole di dati provengono da diversi sindacati, ma non ci informano circa il numero dei loro associati55.

54 Durante la realizzazione delle ricerche della Comissão Nacional da Verdade, la ricercatrice San Romanelli e la dottoranda Heliene Nagasava hanno trovato negli archivi del Ministério do Trabalho e Emprego (MTE), questa documentazione che è stata digitalizzata e costituisce il materiale d'analisi di questo studio. Questo materiale non rappresenta che solo una parte del totale dei documenti sindacali. Tuttavia, ad oggi, rappresenta la totalità del materiale a cui abbiamo accesso, dal momento che la documentazione del MTE si trova in un pessimo stato di conservazione e non è in alcun modo inventariata.

55 In uno studio futuro, che verrà completato nel corso del 2016, intendiamo identificare il numero di associati per ciascuna delle organizzazioni identificate, per poter comprovarela conclusione di Erickson secondo cui i sindacati maggiori furono i più colpiti subito dopo il Golpe. 


\section{Le schede sui sindacati: ripartizione geografica}

Sindacati dello Stato Sindacati di Guanabara Sindacati dello Stato di di Rio de Janeiro São Paulo

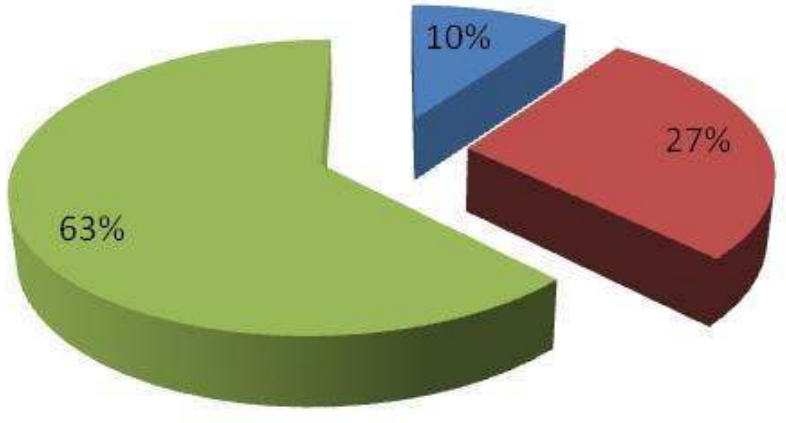

Fig. 1. Ripartizione geografica delle schede sui sindacati.

Sul totale dei sindacati registrati negli Stati di Rio de Janeiro e Guanabara ${ }^{56}$, circa il 17\% subirono un intervento statale durante la dittatura brasiliana. Ma nello Stato di Rio de Janeiro e Guanabara, il 33\% dei sindacati presi in esame subirono un'ingerenza statale (anche precedentemente); nello Stato di São Paulo, il 42\%. Questo dimostra come questa documentazione dica molto anche riguardo ai sindacati che non subirono l'ingerenza statale durante questo periodo, offrendoci così l'opportunità di osservare il funzionamento dell'apparato sindacale quando è controllato dallo Stato.

La maggior parte degli interventi statali sui sindacati si concentrò nel biennio 19641965 e nominò giunte governative al posto di delegati sindacali. Generalmente l'intervento avveniva con l'intento di realizzare nuove elezioni per la regolarizzazione delle attività sindacali e con il pretesto di «accertate irregolarità, sussistendo violazioni delle norme di legge che disciplinano il funzionamento delle organizzazioni sindacali». In altri casi, meno frequenti, la giunta o l'interventor veniva incaricato anche di fornire le prove necessarie per l'incriminazione dei dirigenti sindacali che avevano infranto le rigorose norme stabilite dal regime militare. Come è già stato detto, i rapporti annuali erano obbligatori e un ritardo nella loro presentazione poteva costituire un motivo per un intervento nell'organizzazione sindacale.

${ }^{56}$ Benché il totale delle schede corrisponda a 133 organizzazioni, 49 di queste non registravano le attività degli anni 1964 e 1965, su cui si sofferma questa analisi, e per questa ragione sono state scartate. Nella nostra analisi, pertanto, si è soffermata su 84 organizzaizoni sindacali dello Stato di Rio de Janeiro e Guanabara. 
Si sono verificati casi, come quello del Sindacato dei lavoratori dell'industria edile e dei beni immobili di São José do Rio Preto, in cui il presidente della giunta governativa nominato era già nel direttivo prima del Golpe, avendo fatto parte del consiglio fiscale ed essendo stato delegato sindacale. Notiamo, ugualmente, che membri della giunta governativa rimasero all'interno delle organizzazioni, dopo essere stati regolarmente eletti, proseguendo i loro mandati nel ruolo di interventores.

Troviamo registri come quello del Sindacato dei lavoratori agricoli di São José do Rio Pardo, in cui si richiede il riconoscimento al Ministero alla fine di aprile del 1964, successivamente all'instaurazione del regime militare e la sua concessione viene stabilita nello stesso giorno in cui si verifica l'intervento statale, secondo le Ordinanze n. 139 e n. 231 del 24/04/1964 e 06/07/1964. Dal momento che l'organizzazione dei lavoratori agricoli si integrò solo tardivamente nella struttura sindacale ufficiale, possiamo verificare, soprattutto per i sindacati agricoli dello Stato di São Paulo, che molti direttivi eletti tra il 1962 e il 1963 vennero destituiti immediatamente dopo il golpe e venne decretato un intervento sul sindacato. Alcuni autori sottolineano che nel periodo del governo Goulart, nelle campagne, comunisti e circulistas si disputavano il controllo delle organizzazioni di rappresentanza dei lavoratori rurali. Stando così le cose, a prescindere dalle schede sindacali, che non ci indicano l'appartenenza ideologica dei direttivi, possiamo dedurre che i sindacati su cui si intervenne erano guidati da lavoratori comunisti o sotto quest'area di influenza, che suscitavano i sospetti dei militari al potere.

Affinando l'analisi e facendo una supposizione sul complesso delle intervenções, anche se molte schede non ci informano sulla composizione dei direttivi prima del 1964, constatiamo una rottura causata dal regime militare per allontanare - sia revocando mandati, sia imprigionando i dirigenti - i membri dei direttivi sino ad allora in carica, con ogni probabilità provenienti dalle liste dell'alleanza comunistatrabalhista. Tuttavia, impressiona ugualmente il numero di direttivi $(35,5 \%)$ che rimasero in carica nelle rispettive organizzazioni dopo le prime elezioni tenutesi al termine della dittatura.

Così si presentano nuove domande: che cosa avvenne nei sindacati in cui vi fu continuità nei direttivi? Anche questi dirigenti sindacali dovevano sottomettersi alle nuove esigenze imposte dal regime autoritario? Come hanno fatto a mantenere questa carica all’interno dell'organizzazione per più mandati consecutivi? Furono i dirigenti a evitare che si verificassero interventi da parte dello stato? A queste e ad altre questioni bisognerà dare risposta in uno sviluppo futuro di questa ricerca. 
L'intensità con cui venne portata avanti la repressione contro la classe lavoratrice ha fatto definire a Mattos57 il periodo dal 1964 al 1967 come quello dell'imbavagliamento delle organizzazioni sindacali. Dopo aver ottenuto il controllo dei sindacati, attraverso le intervenções, il governo si impegnò nel rafforzamento del sistema corporativo, stimolando fortemente la sindacalizzazione, accompagnando una politica di compressione salariale con il controllo dell'inflazione. Il carattere assistenzialista del sindacato divenne attrattivo per nuovi gruppi di lavoratori e per $\mathrm{i}$ loro dirigenti sindacali, i cosiddetti "pelegos" (zerbini), che ottenevano una serie di benefit, come ha sottolineato Erickson:

una via preferenziale indirizzata verso il servizio pubblico in caso di disoccupazione, per il credito presso il Banco Nacional de Habitação finalizzato all'acquisto di una casa di proprietà, per la suddivisione e la distribuzione delle proprietà terriere dello Stato, per il finanziamento dei veicoli o degli strumenti utili all'esercizio della propria professione, nell'acquisto o nella locazione di un appartamento passato sotto controllo statale a seguito di una decisione giudiziaria, posti vacanti nel servizio portuale, borse di studio per l'educazione secondaria o l'apprendistato tecnico, per loro o per i loro figli58.

Si verificò uno svuotamento del sindicato e, disprezzando le rivendicazioni politiche ed economiche, un investimento nell'ampliamento del settore assistenziale e del patrimonio fisico delle organizzazioni, avvalendosi delle ingenti somme raccolte con il versamento delle quote associative sindacali.

\section{Considerazioni finali}

A partire dal 1965, Rodrigues59 evidenzia un mutamento nelle ragioni che giustificavano queste misure. Se nel biennio 1964-1965 riscontriamo una dura persecuzione in ambito sindacale, con la principale accusa di "sovversivismo", nel periodo che va dal 1966 al 1970, oltre a rinvenire una diminuzione dell'ingerenza statale - dovuta al controllo statale, conseguito nel periodo precedente, sulla maggioranza delle organizzazioni sindacali -, vennero addotte nuove giustificazioni alla base delle

57 MATTOS, Marcelo Badaró, op. cit.

${ }^{8}$ ERICKSON, Kenneth Paul, op. cit., p. 211.

59 RODRIGUES, Leôncio Martins, Sindicalismo e classe operária (1930-1964), in FAUSTO, Boris (Org.), História geral da civilização brasileira: O Brasil republicano: sociedade e política (1930-1964), v. 10, t. III, São Paulo, DIFEL, 1986, pp. 509-555. 
intervençoes: irregolarità nelle elezioni, mancata approvazione dei rendiconti del direttivo precedentemente in carica, infrazione delle norme e dei regolamenti, per citarne solo alcune.

Questa fase viene definita da Mattos ${ }^{60}$ come un momento di liberalizzazione progressiva delle attività sindicali da parte del Ministero del Lavoro, dal momento che andarono incrementando le elezioni e la costituzione di movimenti intersindacali contrari alla politica di compressione salariale in diverse organizzazioni61; questo emerse con la vittoria di alcuni dirigenti legati alle commissioni per il luogo di lavoro e a partiti di sinistra, soprattutto i soppressi $\mathrm{PCB}$ e $\mathrm{PTB}^{62}$, l'alleanza comunistatrabalhista egemonica nel periodo anteriore al Golpe.

Questo contesto che si manifestò con mobilitazioni e scioperi, riunendo un numero significativo di lavoratori - risaltano Contagem ${ }^{63}$ e Osasco ${ }^{64}$, nel 1968, per citare solamente gli esempi più noti - diede luogo a una nuova onda di intervenções e arresti di leaders sindacali. Il compito che si prospetta di qui in avanti è quello di indagare nel contesto successivo - dal 1966 al dicembre 1968 - per quel che riguarda le intervençoes sindacali. La continuità dell'indagine permetterà l'avanzamento della ricerca su questo tema.

I lavoratori e la loro leadership continuarono a costituire il gruppo sociale maggiormente toccato, monitorato e perseguitato durante la totalità del periodo della dittatura.

Negli anni Settanta il governo idealizzò i sindacati come organi integrati nel sistema ufficiale di previdenza e assistenza sociale, svuotando l'organizzazione di qualsiasi connotazione politica. I sindacati servivano, secondo questa logica, per veicolare le "conquiste" del regime e, in questo contesto, gli interventores avevano un ruolo centrale nella mediazione tra Stato e classe lavoratrice. Solamente alla fine degli anni

60 MATTOS, Marcelo Badaró, op. cit.

${ }^{61}$ Possiamo citare l'esempio più conosciuto del Movimento Intersindical contra o Arrocho Salarial (MIA), formatosi a partire dalla Oposição Sindical Metalúrgica de Osasco, che riuscì a sconfiggere l'interventor nominato in occasione delle elezioni del 1968. Questo aveva la propria base nel lavoro della commissione di fabbrica della Cobrasma e nell'Oposição Sindical Metalúrgica di San Paolo, che, benché fosse stata sconfitta nella competizione elettorale, mise in discussione l'egemonia degli interventores instaurati ai vertici sindacali.

62 Militanti provenienti dal PCB in alleanza con trabalhistas privilegiarono una linea di mobilitazione moderata per non perdere le loro cariche dirigenziali.

${ }_{63} \mathrm{Nel}$ marzo 1968 la presenza di almeno duemila lavoratori dell'industria mineraria tenne a battesimo la creazione del Comitê Intersindical Anti-arrocho di Minas Gerais. Il 16 aprile iniziò uno sciopero che aveva alla base una rivendicazione salariale (l'aumento del $25 \%$ degli stipendi). Lo scioperò parti da Contagem, uno dei principali centri industriali dello Stato e si estese rapidamente fino a coinvolgere quasi 20.000 operai [N.d.T.].

64 L'occupazione della fabbrica metallurgica Cobrasma di Osasco, nello Stato di San Paolo, ebbe inizio il 16 luglio 1968 e coinvolse 3.00o lavoratori. Lo sciopero era stato attentamente pianificato nei dettagli dai lavoratori [N.d.T.]. 
Settanta e con l'incentivo delle aperture politiche, ebbe inizio una nuova fase di affermazione delle organizzazioni collettive dei lavoratori, marcata dai grandi scioperi di massa dell' $A B C$ paulista e di diverse altre regioni brasiliane. Vale la pena evidenziare come i periodi di fermento del movimento dei lavoratori furono spesso accompagnati da una risposta repressiva da parte dello Stato dittatoriale, finendo per intervenire quasi sempre nell'azione dei sindacati più attivi e destituendone i dirigenti. 


\section{* Gli autori}

Alejandra Estevez è ricercatrice post-doc del Centro de Pesquisa e Documentação de História Contemporânea do Brasil della Fundação Getúlio Vargas (CPDOC/FGV) ed è titolare di una borsa di CAPES. Nel 2014 è stata ricercatrice per conto della fondazione Getúlio Vargas sul tema della Repressione dei lavoratori e del movimento sindacale all'interno della Comissão Nacional da Verdade (CNV). Si è addottorata in Sociologia e laureata in Storia sociale presso l'Universidade Federal do Rio de Janeiro (UFRJ).

URL: < http://www.studistorici.com/progett/autori/\#Estevez >

Marco Antonio Teixeira è dottorando in Sociologia dell'Instituto de Estudos Sociais e Políticos dell'Universidade do Estado do Rio de Janeiro (IESP/UERJ), borsista della Fundação Carlos Chagas Filho de Amparo à Pesquisa do Estado do Rio de Janeiro (FAPERJ). Ha conseguito la laurea specialistica in Scienze sociali nell'ambito del corso di studio in Desenvolvimento, Agricultura e Sociedade dell'Universidade Federal Rural do Rio de Janeiro (CPDA/UFRRJ), dopo aver ottenuto una laurea in Scienze sociali presso la UERJ e in Storia presso l'Universidade Federal do Estado do Rio de Janeiro (UNIRIO)

URL: < http://www.studistorici.com/progett/autori/\#Teixeira >

\section{Per citare questo articolo:}

ESTEVEZ, Alejandra, TEIXEIRA, Marco Antônio, «Le conseguenze del golpe del 1964 sul movimento sindacale brasiliano», Diacronie. Studi di Storia Contemporanea : Le dittature militari: fisionomia ed eredità politica, 29/12/2015,

URL:<http://www.studistorici.com/2015/12/29/estevez-teixeira_numero_24/ >

Diacronie Studi di Storia Contemporanea 0 www.diacronie.it

Risorsa digitale indipendente a carattere storiografico. Uscita trimestrale. redazione.diacronie@hotmail.it

Comitato di redazione: Jacopo Bassi - Luca Bufarale - Elisa Grandi - Antonio César Moreno Cantano - Deborah Paci - Fausto Pietrancosta - Alessandro Salvador - Matteo Tomasoni - Luca Zuccolo

Diritti: gli articoli di Diacronie. Studi di Storia Contemporanea sono pubblicati sotto licenza Creative Commons 3.0. Possono essere riprodotti e modificati a patto di indicare eventuali modifiche dei contenuti, di riconoscere la paternità dell'opera e di condividerla allo stesso modo. La citazione di estratti è comunque sempre autorizzata, nei limiti previsti dalla legge. 